\title{
Postoperative sufentanil intravenous patient-controlled analgesia within the first 24 hours: a retrospective study
}

\author{
Weishan Lee, Xiang Gao, Jie Tang, Ailun Li, Yiqi Zhu, Xiaomin Ling, Jing Cang, Fang Fang \\ Department of Anesthesia, Zhongshan Hospital, Fudan University, Shanghai, China \\ Contributions: (I) Conception and design: W Lee, X Gao, F Fang, J Cang; (II) Administrative support: F Fang, J Cang; (III) Provision of study \\ materials or patients: F Fang, J Cang; (IV) Collection and assembly of data: W Lee, X Gao, J Tang, A Li, Y Zhu, X Ling; (V) Data analysis and \\ interpretation: W Lee, X Gao; (VI) Manuscript writing: All authors; (VII) Final approval of manuscript: All authors. \\ Correspondence to: Fang Fang; Jing Cang. Department of Anesthesia, Zhongshan Hospital, Fudan University, 180 Fenglin Road, Xuhui District, \\ Shanghai 200032, China. Email: jerryfang81@hotmail.com; cangjing_zs@sina.com.
}

Backgroundk Intravenous patient-controlled analgesia (IV-PCA) is recommended for postoperative
systemic analgesia by the American Pain Society. As there is no efficacy advantage and a higher probability
of adverse events, routine basal infusion of opioids is not recommended for opioid-naïve adults. However,
the opioids referred to in postoperative pain management guidelines were mainly morphine. Nowadays,
sufentanil is widely used in postoperative acute pain management. In this retrospective study, we evaluated
and compared the analgesic effect, PCA use, as well as adverse events among different basal infusions with
sufentanil-based postoperative PCA. Methods: The data of 322 eligible postoperative patients who received sufentanil-based IV-PCA from January 2018 to December 2019 were collected in this study. According to the settings of background infusions, patients were allocated to 3 groups: 2 , 1 , or $0.5 \mathrm{~mL} /$ hour. The primary endpoint was PCA attempts and successful delivery. We also evaluated the occurrence of adverse events associated with sufentanil-based PCA and the intensity of postoperative pain using the Numeric Rating Scale (NRS).

Results: PCA attempts, successful deliveries, total volume of PCA and patient NRS scores were significantly different between the 3 groups $(\mathrm{P}<0.05)$. Through pairwise comparison, there was only a statistical difference between the $2 \mathrm{~mL} /$ hour and the $0.5 \mathrm{~mL} /$ hour group in PCA attempts, successful deliveries, and total volumes of PCA. There were no statistical differences in adverse events between groups $(\mathrm{P}>0.05)$.

Conclusions: We found that a smaller background infusion with sufentanil required more bolus infusions and a higher total volume of PCA within 24 hours after surgery. However, NRS scores were higher in the smaller background infusion group. Our results highlight the need for further studies to optimize doses for sufentanil IV-PCA basal infusions, which will also provide useful information to enhance the quality of pain control in the future.

Keywords! Patient-controlled analgesia (PCA), sufentanil, basal infusion, postoperative pain

Submitted Aug 24, 2020. Accepted for publication Nov 18, 2020.

doi: 10.21037/apm-20-1939

View this article at: http://dx.doi.org/10.21037/apm-20-1939

\section{Introduction}

Approximately $75 \%$ of patients experience undesirable postoperative pain (1). Inadequate pain management leads to delayed recovery, chronic postsurgical pain, and poor quality of life. In 2012 and 2016, the American Society of
Anesthesiologists (ASA) and the American Pain Society individually published guidelines for postoperative pain management (2), however, successful postoperative analgesia remains a challenge $(3,4)$.

Patient-controlled analgesia (PCA) allows the 


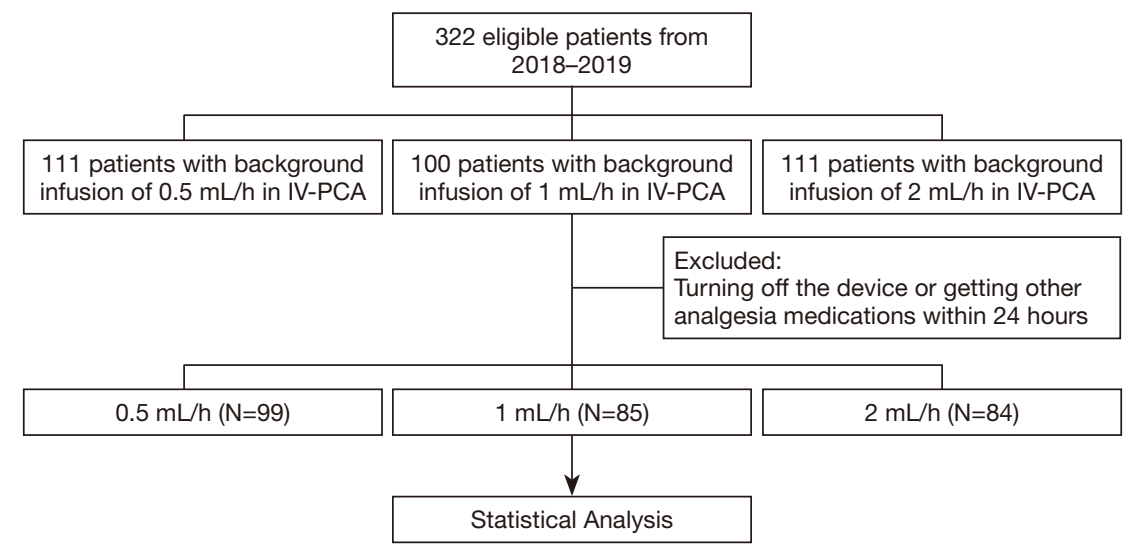

Figure 1 Flow diagram of the study design. IV-PCA, intravenous patient-controlled analgesia.

control of pain relief to be transferred to patients, and is recommended as a primary pain control method. Compared to conventional methods, such as healthcare provider-initiated bolus dosing, PCA demonstrates greater effectiveness and patient satisfaction, and similar incidences of opioid-related adverse events such as nausea, sedation, or respiratory depression (5-7). As there is no efficacy advantage and a higher probability of adverse events, guidelines for perioperative pain management from both the ASA and the American Pain Society do not recommend the addition of a background infusion $(2,6,8)$. However, after closely looking into the references used in these guidelines, we found that the opioids involved were mainly morphine $(9,10)$. The only meta-analysis showing an increased risk of nausea and vomiting, and even increased risk of respiratory depression in adults listed different opioid analgesics (11).

Due to its enhanced analgesic efficacy and lower incidence of adverse effects compared to morphine, fentanyl is among the first choices for postoperative analgesics (12). Its analog, sufentanil, acts selectively at the opioid receptor with a higher therapeutic index and lower frequency of respiratory suppression than fentanyl $(13,14)$. Moreover, most sufentanil is catalyzed by the cytochrome P450 enzyme system to inactive metabolites, while a very low fraction is metabolized to active metabolites with $10 \%$ of the activity of sufentanil (15). Sufentanil is used in target-controlled infusion (TCI), as it is believed that intravenous PCA with a basal infusion has a steady effectsite concentration compared to a PCA bolus dose alone. A previous study also showed that basal infusion was safe and with a very low incidence of adverse events with sufentanil (16). However, there was no evidence showing the effects of different basal infusions on pain control and sufentanil sparing.

In this retrospective study, we compared 3 different basal infusions of sufentanil to investigate the efficacy, safety and drug sparing of sufentanil-based intravenous patient-controlled analgesia (IV-PCA) with basal infusion. We present the following article in accordance with the STROBE reporting checklist (available at http://dx.doi. org/10.21037/apm-20-1939).

\section{Methods}

\section{Study design and patient selection}

This was a retrospective, single center, controlled clinical study. The data of 322 postoperative patients aged between 25 and 80 years who received general anesthesia with IV-PCA based on sufentanil for postoperative pain management in Zhongshan Hospital Affiliated to Fudan University from January 2018 to December 2019 were collected. According to the setting of the background infusion, patients were allocated to 3 groups: 2 , 1 , or $0.5 \mathrm{~mL} /$ hour. The study design and process is described in the diagram below (Figure 1). The study was conducted in accordance with the Declaration of Helsinki (as revised in 2013). The study was approved by The Ethics Committee of the Zhongshan Hospital, Fudan University (No. B2019199R), and written informed consent was obtained from all patients.

Patients were excluded from study if they had perioperative abnormal liver function, regional anesthesia or analgesia, the use of intravenous PCA for less than 
Table 1 Patient demographic characteristics

\begin{tabular}{|c|c|c|c|c|}
\hline Parameters & $2 \mathrm{~mL} /$ hour $(\mathrm{N}=84)$ & $1 \mathrm{~mL} /$ hour $(\mathrm{N}=85)$ & $0.5 \mathrm{~mL} /$ hour $(\mathrm{N}=99)$ & $P$ value \\
\hline Gender (male/female) & $43 / 41$ & $40 / 45$ & $52 / 47$ & 0.748 \\
\hline Weight (kg) & $66.8 \pm 11.54$ & $64.46 \pm 9.01$ & $66.89 \pm 11.96$ & 0.355 \\
\hline Height (cm) & $166.12 \pm 7.25$ & $165.88 \pm 7.66$ & $166.06 \pm 7.71$ & 0.895 \\
\hline Surgical procedures, $\mathrm{n}(\%)$ & & & & 0.33 \\
\hline Gynecological surgery & $11(13.1)$ & $14(16.46)$ & $8(8.08)$ & \\
\hline General surgery & $32(38.1)$ & $23(27.06)$ & $31(31.31)$ & \\
\hline Thoracic surgery & $17(20.24)$ & $21(24.71)$ & $20(20.2)$ & \\
\hline
\end{tabular}

24 hours, taking other non-opioid analgesia medications after surgery (due to routine use from certain surgeons), or accompanying surgical or anesthetic complications.

Demographic characteristics including age, sex, weight, height, body mass index (BMI) and surgical procedures were collected from the patients' medical records. To measure the intensity of postoperative pain at rest and during movement, the Numeric Rating Scale (NRS, 0-10) was recorded on day 1 after surgery. The frequency of unsatisfied demands was recorded and the ratio between the number of successfully delivered doses to the number of attempts were calculated. The opioid-related adverse events, such as nausea, vomiting, dizziness, itching, hypotension, and respiratory depression were also recorded.

The primary outcome of this study was to compare PCA attempts and successful delivery. The secondary outcome of the study was to compare the adverse events associated with opioids in PCA.

\section{Analgesia management}

All patients received general anesthesia followed by intravenous PCA with sufentanil after surgery. After the end of surgery, patients were transferred to a post-anesthesia care unit (PACU) where intravenous PCA was initiated. Intravenous PCA was performed with sufentanil $1 \mu \mathrm{g} / \mathrm{mL}$ and ramosetron $0.6 \mathrm{mg}$ mixed with $0.9 \%$ isotonic saline with a lockout period of $8-10$ minutes, and a single bolus injection volume of 2-4 mL. According to anesthesiologists' own considerations, continual basal infusion was set at a rate of $0.5,1$, or $2 \mathrm{~mL} /$ hour.

\section{Statistical analysis}

The SPSS 22.0 statistical software was used for statistical processing. All data were presented as mean \pm standard deviation or counts with percentages. Comparisons between groups were compared using a Student's $t$-test or a chi-square test. A normality test was conducted before these statistical tests. Statistical significance was set at $\mathrm{P}<0.05$.

\section{Results}

A total of 322 patients received intravenous PCA with sufentanil after surgery during the study period. Among these patients, 54 were excluded due to the device being turned off, or they received other analgesia medications within 24 hours. The demographic characteristics of the patients are presented in Table 1. There were no statistical differences in age, height, weight, gender, or surgical procedures between the groups.

The PCA-related variables of the 3 groups are shown in Table 2. PCA attempts, successful deliveries, and total volumes of PCA were significantly different between the 3 groups $(\mathrm{P}<0.05)$. However, through pairwise comparison, there was only a statistical difference between the 2 and $0.5 \mathrm{~mL}$ /hour group. Furthermore, patient NRS scores in the smaller continual basal infusion group were significantly higher than the $2 \mathrm{~mL} /$ hour group $(\mathrm{P}<0.05)$.

There was no statistical difference in adverse events between groups (Table 3). All patients with opioid-related adverse events recovered after conservative management. 
Table 2 The sufentanil-based PCA-related variables between different background infusions on day 1 following surgery

\begin{tabular}{|c|c|c|c|c|}
\hline Parameters & $2 \mathrm{~mL} /$ hour $(\mathrm{N}=84)$ & $1 \mathrm{~mL} /$ hour $(\mathrm{N}=85)$ & $0.5 \mathrm{~mL} / \mathrm{hour}(\mathrm{N}=99)$ & $P$ value \\
\hline PCA successful delivery & $2.95 \pm 3.53$ & $4.72 \pm 5.43$ & $5.2 \pm 5.11^{\mathrm{a}}$ & $0.005^{*}$ \\
\hline PCA successful delivery/attempt & $0.92 \pm 0.17$ & $0.9 \pm 0.22$ & $0.89 \pm 0.21$ & 0.244 \\
\hline Total volumes of PCA $(\mu \mathrm{g})$ & $11 \pm 13.38$ & $17.41 \pm 20.6$ & $18.95 \pm 18.39^{a}$ & $0.006^{*}$ \\
\hline Mean NRS score during movement & $2.46 \pm 1.59$ & $3.64 \pm 1.68^{\mathrm{a}}$ & $3.4 \pm 1.63^{\mathrm{a}}$ & $<0.001^{*}$ \\
\hline
\end{tabular}

${ }^{a}, \mathrm{P}<0.05$ vs. $2 \mathrm{~mL} /$ hour; ${ }^{*}$, significant $(\mathrm{P}<0.05)$. PCA, patient-controlled analgesia; NRS, Numeric Rating Scale.

Table 3 The opioid-related adverse events of sufentanil-based intravenous PCA

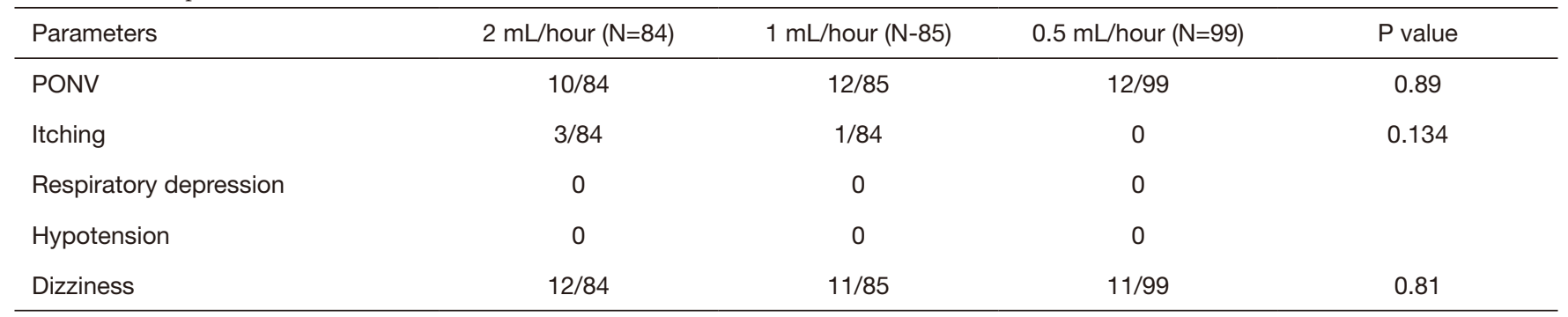

PCA, patient-controlled analgesia; PONV, postoperative nausea and vomiting.

\section{Discussion}

In this study, we showed that sufentanil IV-PCA with different basal infusions was safe and effective in postoperative pain management. In addition, relatively higher basal infusions demonstrated significant total sufentanil sparing within 24 hours.

For postoperative acute pain, conventional treatments relied on the intermittent use of analgesics administered by a healthcare provider. PCA then became the first choice in postoperative pain management because of its greater effectiveness and higher patient satisfaction $(6,7)$.

According to the guidelines from the ASA and American Pain Society, basal infusion is not recommended in IVPCA with opioids for opioid-naïve patients. However, most of references they used in these guidelines were from morphine IV-PCA. Morphine is not suitable for continuous infusion because of its long action time and powerful active metabolite morphine-6-glucuronide. Nowadays, sufentanil is widely used in postoperative acute pain management because of its higher therapeutic index and lower frequency of respiratory suppression. However, little was known about its IV-PCA properties, efficacy, and drug sparing, especially with basal infusion. In our study, we found that patients with smaller continual basal infusions showed greater total sufentanil consumption within 24 hours after surgery. Furthermore, higher basal infusions demonstrated more effective analgesia and a similar adverse event profile.

Opioid metabolites should be considered when it comes to the use of basal infusion and other settings of IV-PCA. For example, pethidine is metabolized to norpethidine. After high-dose pethidine intake, the toxic reaction results in anxiety, nervousness, twitching, tremors and even seizures $(17,18)$. Patients with renal malfunction are suited to agents with no active metabolites, such as fentanyl (19). Within a standard 8-10 minutes lockout period as used in PCA, a bolus dose of hydromorphone reaches its peak effect easier, whereas an equivalent morphine bolus dose would not. Thus, it would be possible to stack morphine doses with PCA, leading to more side effects because of relative overdose (20,21). Different to morphine, a basal infusion is suitable in IV-PCA with sufentanil because of its short halflife of clearance (22) and higher therapeutic index compared with other opioids (23-25). The safety of basal infusions with sufentanil was also confirmed by other studies (26).

It is believed that the effect of IV-PCA is independent of the agent used, whether high or low potency, and it is 
more likely to be affected by the settings of parameters (27). There are no major differences in efficacy or opioid-related side effects between morphine and other commonly used opioids such as pethidine (28), hydromorphone (29-31), and fentanyl, although pruritus may be seen with morphine use. In this study, the efficacy of analgesia represented by NRS scores showed that the smaller basal infusion of sufentanil was greater not only at rest but also during movement. Our results indicated that sufentanil IV-PCA with a $2 \mathrm{ml} /$ hour basal infusion was more effective in postoperative pain management.

Previous research has shown that high dose sufentanil basal infusion in IV-PCA had a greater chance of relieving pain upon activity with no additional adverse events (10). However, the total amount of sufentanil did not record. In our study, the total amounts in the high basal infusion group were less than that in low basal infusion group. This indicated a potential drug sparing effect, which is a key element in enhanced recovery after surgery (ERAS). Contrary to previous research (10), patients in our study did not demand rescue analgesia because the bolus dose in this study was higher.

In conclusion, sufentanil IV-PCA with relatively higher basal infusion showed greater efficacy in postoperative pain management, comparable incidence of adverse events, and a potential drug sparing effect.

As a retrospective and single-center study, this study had some limitations. First, because of guideline recommendations, the amount of cases receiving more than $1 \mu \mathrm{g} /$ hour basal infusion was limited at our center. Second, all the data was collected from the work of the Acute Pain Service (APS) group who performed the follow-up only once within 24 hours postoperatively. More well-designed randomized studies are needed to further evaluate the effect of different basal infusions of PCA.

\section{Acknowledgments}

Funding: None.

\section{Footnote}

Reporting Checklist: The authors have completed the STROBE reporting checklist. Available at http://dx.doi. org/10.21037/apm-20-1939

Data Sharing Statement: Available at http://dx.doi. org/10.21037/apm-20-1939
Conflicts of Interest: All authors have completed the ICMJE uniform disclosure form (available at http://dx.doi. org/10.21037/apm-20-1939). The authors have no conflicts of interest to declare.

Ethical Statement: The authors are accountable for all aspects of the work in ensuring that questions related to the accuracy or integrity of any part of the work are appropriately investigated and resolved. The study was conducted in accordance with the Declaration of Helsinki (as revised in 2013). The study was approved by The Ethics Committee of the Zhongshan Hospital, Fudan University (No. B2019-199R), and written informed consent was obtained from all patients.

Open Access Statement: This is an Open Access article distributed in accordance with the Creative Commons Attribution-NonCommercial-NoDerivs 4.0 International License (CC BY-NC-ND 4.0), which permits the noncommercial replication and distribution of the article with the strict proviso that no changes or edits are made and the original work is properly cited (including links to both the formal publication through the relevant DOI and the license). See: https://creativecommons.org/licenses/by-nc-nd/4.0/.

\section{References}

1. Mercieri M, D'Andrilli A, Arcioni R. Improving postoperative pain management after video-assisted thoracic surgery lung resection contributes to enhanced recovery, but guidelines are still lacking. J Thorac Dis 2018;10:S983-7.

2. American Society of Anesthesiologists. Practice Guidelines for Acute Pain Management in the. Anesthesiology 2012;116:248-73.

3. Pogatzki-Zahn E, Kutschar P, Nestler N, et al. A Prospective Multicentre Study to Improve Postoperative Pain: Identification of Potentialities and Problems. PLoS One 2015;10:70-80.

4. White PF, Kehlet H. Improving postoperative pain management: what are the unresolved issues? Anesthesiology 2010;112:220-5.

5. Tai YH, Wu HL, Lin SP, et al. Influential factors of postoperative pain trajectories in patients receiving intravenous patient-controlled analgesia: a single-centre cohort study in Taiwan. BMJ Open 2019;9:1-7.

6. McNicol ED, Ferguson MC, Hudcova J. Patient controlled opioid analgesia versus non-patient controlled 
opioid analgesia for postoperative pain. Cochrane Database Syst Rev 2015;2015:CD003348.

7. Hudcova J, McNicol E, Quah C, et al. Patient controlled opioid analgesia versus conventional opioid analgesia for postoperative pain. Cochrane Database Syst Rev 2006;(4):CD003348.

8. American Society of Anesthesiologists Task Force on Acute Pain Management. Practice guidelines for acute pain management in the perioperative setting: an updated report by the American Society of Anesthesiologists Task Force on Acute Pain Management. Anesthesiology 2012;116:248-73.

9. Guler T, Unlugenc H, Gundogan Z, et al. A background infusion of morphine enhances patient-controlled analgesia after cardiac surgery. Can J Anaesth 2004;51:718-22.

10. Dal D, Kanbak M, Caglar M, et al. A background infusion of morphine does not enhance postoperative analgesia after cardiac surgery. Can J Anaesth 2003;50:476-9.

11. George JA, Lin EE, Hanna MN, et al. The effect of intravenous opioid patient-controlled analgesia with and without background infusion on respiratory depression: A meta-analysis. J Opioid Manag 2010;6:47-54.

12. Stanley TH. The fentanyl story. J Pain 2014;15:1215-26.

13. Bailey PL, Streisand JB, East KA, et al. Differences in magnitude and duration of opioid-induced respiratory depression and analgesia with fentanyl and sufentanil. Anesth Analg 1990;70:8-15.

14. van de Donk T, Ward S, Langford R, et al. Pharmacokinetics and pharmacodynamics of sublingual sufentanil for postoperative pain management. Anaesthesia 2018;73:231-7.

15. Tateishi T, Krivoruk Y, Ueng YF, et al. Identification of human liver cytochrome P-450 3A4 as the enzyme responsible for fentanyl and sufentanil $\mathrm{N}$-dealkylation. Anesth Analg 1996;82:167-72.

16. Zhen L, Li X, Gao X, et al. Dose determination of sufentanil for intravenous patient-controlled analgesia with background infusion in abdominal surgeries: A random study. PLoS One 2018;13:1-11.

17. Stone PA, Macintyre PE, Jarvis DA. Norpethidine toxicity and patient controlled analgesia. Br J Anaesth 1993;71:738-40.

18. Kussman BD, Sethna NF. Pethidine-associated seizure in a healthy adolescent receiving pethidine for postoperative pain control. Paediatr Anaesth 1998;8:349-52.

19. Macintyre PE. Intravenous patient-controlled analgesia: One size does not fit all. Anesthesiol Clin North America 2005;23:109-23.

20. Hong D, Flood P, Diaz G. The side effects of morphine and hydromorphone patient-controlled analgesia. Anesth Analg 2008;107:1384-9.

21. Felden L, Walter C, Harder S, et al. Comparative clinical effects of hydromorphone and morphine: A meta-analysis. Br J Anaesth 2011;107:319-28.

22. Oh SK, Lee IO, Lim BG, et al. Comparison of the analgesic effect of sufentanil versus fentanyl in intravenous patient-controlled analgesia after total laparoscopic hysterectomy: A randomized, double-blind, prospective study. Int J Med Sci 2019;16:1439-46.

23. Momeni M, Crucitti M, De Kock M. Patient-controlled analgesia in the management of postoperative pain. Drugs 2006;66:2321-37.

24. Schug SA, Torrie JJ. Safety assessment of postoperative pain management by an acute pain service. Pain 1993;55:387-91.

25. Wheeler M, Oderda GM, Ashburn MA, et al. Adverse events associated with postoperative opioid analgesia: A systematic review. J Pain 2002;3:159-80.

26. Gao Y, Deng X, Yuan H, et al. Patient-controlled Intravenous Analgesia with Combination of Dexmedetomidine and Sufentanil on Patients after Abdominal Operation A Prospective, Randomized, Controlled, Blinded, Multicenter Clinical Study. Clin J Pain 2018;34:155-61.

27. Macintyre PE. Safety and efficacy of patient-controlled analgesia. Br J Anaesth 2001;87:36-46.

28. Doyle E, Robinson D, Morton NS. Comparison of patient-controlled analgesia with and without a background infusion after lower abdominal surgery in children. Br J Anaesth 1993;71:670-3.

29. Rapp SE, Egan KJ, Ross BK, et al. A Multidimensional Comparison of Morphine and Hydromorphone PatientControlled Analgesia. Anesth Analg 1996;82:1043-8.

30. DiGiusto M, Bhalla T, Martin D, et al. Patient-controlled analgesia in the pediatric population: Morphine versus hydromorphone. J Pain Res 2014;7:471-5.

31. Hutchison RW, Eun HC, Tucker WF, et al. A comparison of a fentanyl, morphine, and hydromorphone patientcontrolled intravenous delivery for acute postoperative analgesia: A multicenter study of opioid-induced adverse reactions. Hosp Pharm 2006;41:659-63.

Cite this article as: Lee W, Gao X, Tang J, Li A, Zhu Y, Ling X Cang J, Fang F. Postoperative sufentanil intravenous patientcontrolled analgesia within the first 24 hours: a retrospective study. Ann Palliat Med 2020;9(6):3932-3937. doi: 10.21037/apm-201939 